\title{
Three-dimensional architecture of pericardial nephrocytes in Drosophila melanogaster revealed by FIB/SEM tomography
}

\author{
Yuto Kawasaki $^{1}$ - Akira Matsumoto $^{2} \cdot$ Takayuki Miyaki $^{1} \cdot$ Mui Kinoshita $^{1} \cdot$ Soichiro Kakuta $^{3} \cdot$ Tatsuo Sakai $^{1}$. \\ Koichiro Ichimura ${ }^{1,3}$ (D)
}

Received: 21 January 2019 / Accepted: 15 April 2019 / Published online: 14 May 2019

(C) The Author(s) 2019

\begin{abstract}
Nephrocytes are similar in structure to podocytes and play a role in the isolation of toxic substances from hemolymph in insects. Drosophila melanogaster nephrocytes have recently been used to study podocyte function and disease. However, the threedimensional ultrastructure of nephrocytes is not clearly understood because their surrounding basement membrane makes it difficult to observe using conventional scanning electron microscopy. We reconstructed the three-dimensional ultrastructure of Drosophila pericardial nephrocytes using serial focused-ion beam/scanning electron microscopy (FIB/SEM) images. The basal surfaces were occupied by foot processes and slit-like spaces between them. The slit-like spaces corresponded to the podocyte filtration slits and were formed by longitudinal infolding/invagination of the basal plasma membrane. The basal surface between the slit-like spaces became the foot processes, which ran almost linearly, and had a "washboard-like" appearance. Both ends of the foot processes were usually anastomosed to neighboring foot processes and thus free ends were rarely observed. We demonstrated that FIB/SEM is a powerful tool to better understand the three-dimensional architecture of nephrocytes.
\end{abstract}

Keywords 3D ultrastructure $\cdot$ Podocytes $\cdot$ Nephrocytes $\cdot$ Drosophila melanogaster $\cdot$ FIB/SEM tomography

\section{Introduction}

Excretion is crucial to maintain the homeostasis of body fluid in metazoans. The metazoan excretory system generally produces primary urine by ultrafiltration of body fluid via filtration epithelia. The primary urine is subsequently transferred to the modulating tubule, where it is modified by epithelial

Electronic supplementary material The online version of this article (https://doi.org/10.1007/s00441-019-03037-3) contains supplementary material, which is available to authorized users.

Koichiro Ichimura

ichimura@juntendo.ac.jp

1 Department of Anatomy and Life Structure, Juntendo University Graduate School of Medicine, 2-1-1 Hongo, Bunkyo-ku, Tokyo 113-8421, Japan

2 Department of Biology, Juntendo University School of Medicine, Inzai, Chiba, Japan

3 Laboratory of Morphology and Image Analysis, Center for Biomedical Research Resources, Juntendo University Graduate School of Medicine, Tokyo, Japan secretion and reabsorption and discharged outside the body as the final urine (Ichimura and Sakai 2017; Ruppert et al. 2003). Filtration epithelia are formed by podocytes in eucoelomates, which include vertebrates (Ruppert and Smith 1988). In vertebrate kidneys, the glomeruli include the podocyte-based filtration epithelia and the renal tubule plays a role as the modulating tubule (Fig. 1a-d) (Ruppert 1994).

Podocytes exhibit a characteristic architecture that is specialized for the ultrafiltration of body fluid (Kriz and Kaissling 2007). The podocyte cell body possesses several thick primary processes, each of which subsequently projects a number of fine foot processes (Fig. 2c). Adjacent podocytes are interdigitated with each other at their foot processes (Fig. 2a-c), which are separated from each other by filtration slits and bridged with a specialized intercellular junction, slit diaphragm, which serves as a filtration barrier apparatus (Figs 1d and 2c) (Pavenstadt et al. 2003).

Pterygote insects, including the fruit fly (Drosophila melanogaster), are the exception in that they do not possess filtration epithelia; instead, they produce urine via epithelial transport of the modulating tubules that are called Malpighian tubules (Fig. 1e) (Kolosov et al. 2018; Sun et al. 2018). 

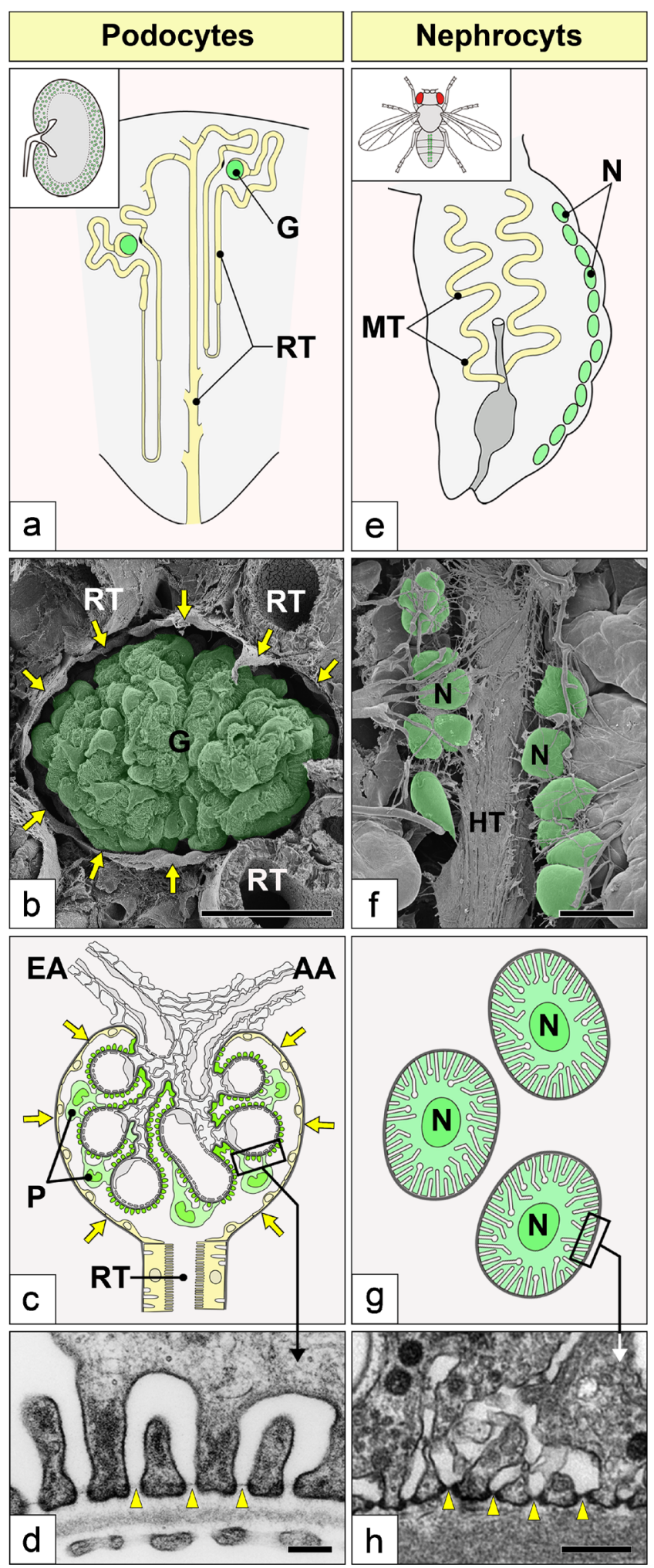

Nephrocytes, which are similar in structure to podocytes, exist in the hemolymph space independently from the Malpighian tubules (Fig. 1e-g) and play a role in the isolation of toxic substances, such as heavy metals, from insect body fluid, hemolymph (Crossley 1984). Nephrocytes form numerous foot
Fig. 1 Localization of podocytes and nephrocytes. (a-d) Podocytes in rat kidney. (a) Schematic drawing of nephrons. Podocytes are localized at the glomerulus $(\mathrm{G})$, a primary urine-producing apparatus. The renal tubule (RT) subsequently receives and modifies the primary urine by epithelial secretion and reabsorption. (b) Conventional scanning electron microscope (SEM) image of a glomerulus. Podocytes form the most outer epithelial layer of the glomerulus (G). RT, renal tubule; arrows, parietal layer of Bowman's capsule. (c) Schematic drawing of a glomerulus. The epithelial layer of podocytes (P) covers the glomerular capillaries and continues to the renal tubule (RT) via a thin parietal layer of the Bowman's capsule (arrows). AA, afferent arteriole; EA, efferent arteriole. (d) Conventional transmission electron microscope (TEM) image of the glomerular wall. Podocytes cover the glomerular basement membrane (GBM) by the foot processes, which are bridged by the slit diaphragm (arrows). (a) and (c) are based on Kriz and Kaissling (2007). (e-h) Pericardial nephrocytes (PCNs) in Drosophila melanogaster. (e) Schematic drawing of PCNs (N) and Malpighian tubules (MT). PCNs exist independently from Malpighian tubules, thus they do not serve as a primary urine-producing apparatus. (f) Conventional SEM of PCNs (N). Multiple PCNs line both sides of the heart tube (HT), which is beneath the dorsal body wall. (g) Schematic drawing of PCNs (N). Each PCN occurred without forming intercellular junctions with other cells since PCNs are individually enwrapped by the basement membrane. (h) Conventional TEM of a PCN. Like podocytes, PCNs formed foot processes and a slit diaphragm (arrowheads) between them. Scale bars: $50 \mu \mathrm{m}$ in (b) and (f); $200 \mathrm{~nm}$ in (d) and (h)

processes that adhere to the basement membrane and possess slit diaphragms between them (Fig. 1h). The molecular components of the slit diaphragm are highly conserved between the nephrocytes in D. melanogaster and podocytes in vertebrates (Weavers et al. 2009; Zhuang et al. 2009) and Drosophila nephrocytes have recently been used as a model system to study podocyte function and disease (Fu et al. 2017; Helmstädter and Simons 2017; $\mathrm{Na}$ et al. 2015; Tutor et al. 2014; Weide et al. 2017).

Fig. 2 Structural similarity and difference in podocytes and nephrocytes. (a-e) Podocytes in rat kidney. (f-j) Pericardial nephrocytes (PCNs) in Drosophila melanogaster. Two neighboring podocytes or PCNs are indicated by different coloring (purple and green). (a, b, f, g) Schematic drawings of two neighboring podocytes $(\mathrm{P})$ or PCNs $(\mathrm{N})$. Podocytes interdigitate each other via the foot processes $(\mathbf{a}, \mathbf{b})$; PCNs exist independently from each other (f) and form the foot processes by basal infolding/ invagination (g). The slit diaphragms are formed between foot processes in podocytes and PCNs $(\mathbf{b}, \mathbf{g})$. The podocyte slit diaphragm is regarded as an intercellular junction; the slit diaphragm of PCNs is regarded as an autocellular junction. The arrowheads indicate the directions of observation in (c)-(e), (h) and (i). E, glomerular endothelial cell; M, mesangial cell. (c, h) Conventional scanning electron microscope (cSEM) image. cSEM is useful to observe the characteristic architecture of podocytes (P) (c); however, it is not impossible to observe the foot processes of PCNs (N) because PCNs are enwrapped by the basement membrane (h). (d, e, i) Reconstructed podocytes or PCN based on serial focused-ion beam/ scanning electron microscopy (FIB/SEM) images. The basal surface structure of podocytes (e) and PCN (i), which cannot be observed by cSEM, is clearly visible in the reconstructed images. (j) Schematic drawing for the orientation of the reconstructed image. The reconstructed podocytes and PCN shown here are also shown in S1 and S2. Scale bar: $1 \mu \mathrm{m}$ 

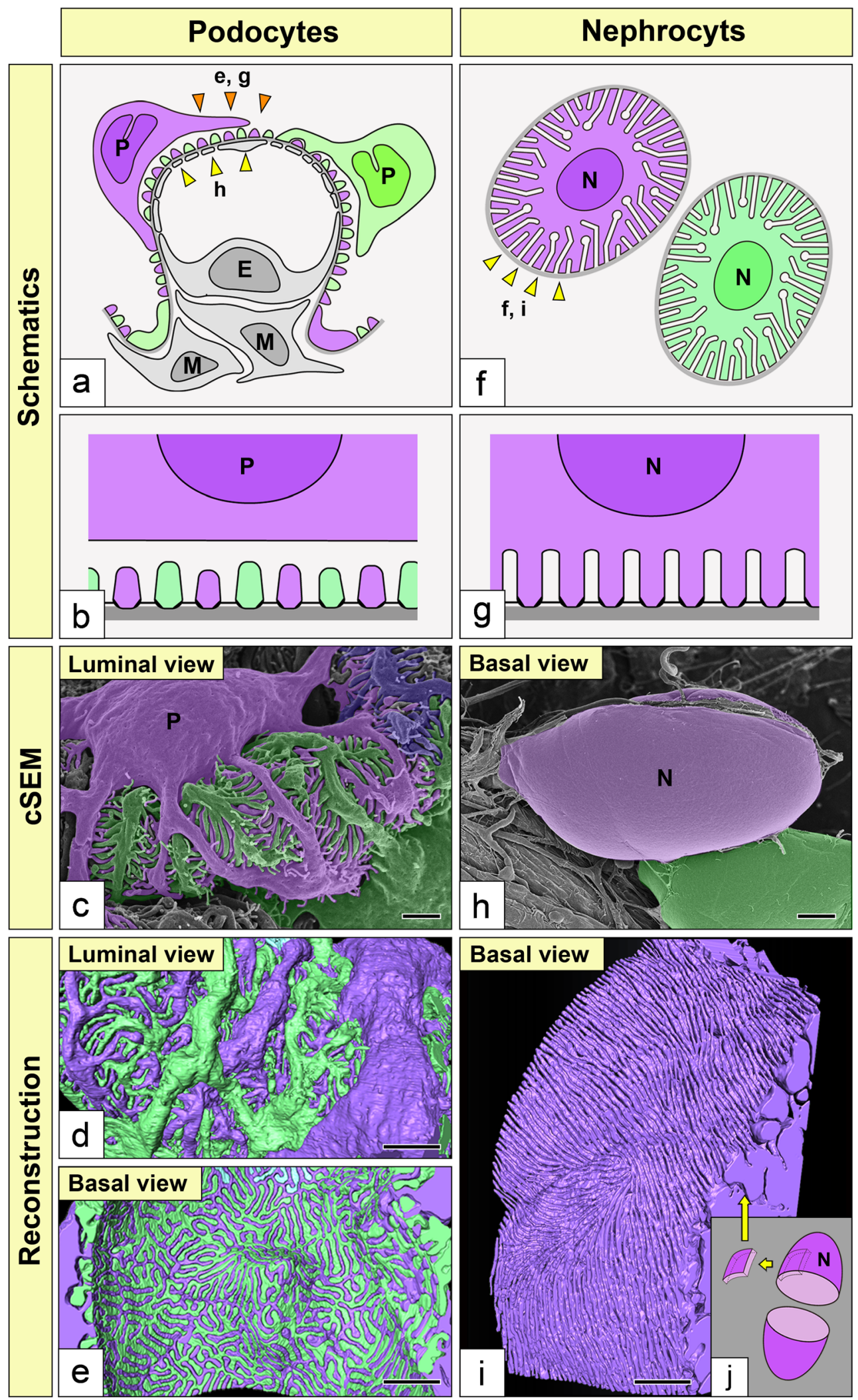

Although the foot processes are similar in structure between Drosophila nephrocytes and vertebrate podocytes, the formation of the foot processes differs greatly between them. Podocyte foot processes are formed by the interdigitation between adjacent podocytes (Fig. 2a-c); thus, the podocyte slit diaphragm is regarded as an intercellular junction. In D. melanogaster, 
individual nephrocytes exist independently without intercellular junctions forming between them (Fig. 2e) and the nephrocyte foot processes are formed by the infolding/invagination of the basal plasma membrane (Fig. 2g). The slit diaphragms of nephrocytes are regarded as autocellular junctions.

Recently, we revealed the three-dimensional (3D) ultrastructural architecture of podocytes using focused ion beam/ scanning electron microscopy (FIB/SEM) tomography in combination with a reconstruction technique (Ichimura et al. 2015, 2017, 2019) (see Fig. S1 for further details of this technique). The reconstructed images of podocytes were similar to the images acquired by conventional SEM (Fig. 2c, d). Furthermore, the reconstructed images clearly visualized the basal surfaces of the podocytes, which are difficult to observe by conventional SEM due to their surrounding basement membrane (Fig. 2e; Movie S1).

In the present study, we reconstructed the ultrastructure of nephrocytes in D. melanogaster using FIB/SEM tomography (Fig. 2i, j) and thus successfully described their 3D architecture using this approach.

\section{Materials and methods}

\section{Drosophila melanogaster}

Drosophila melanogaster cultures and crosses were performed using standard fly food that contained yeast, cornmeal and glucose and the flies were raised at $25^{\circ} \mathrm{C}$. The migrating larvae and 4-week-old adults of Canton-S strain were used to analyze the normal 3D structure of the PCNs. The nephrocyte-specific snsknockdown flies were generated by mating male sns-UAS-RNAi (P\{KK108577\}VIE-260B established at the Vienna Drosophila RNAi Center) and female Dot-GAL4 (Kimbrell et al. 2002) from the Bloomington Drosophila Stock Center. Migrating larvae and adults were fixed in $2.5 \%$ glutaraldehyde solution buffered with $0.1 \mathrm{M}$ phosphate buffer. The fixed samples were further immersed in the same fixative solution and stored at $4{ }^{\circ} \mathrm{C}$.

\section{Rats}

The ultrastructure of podocytes from adult (10-week-old, male) Wistar rats (Charles River Japan, Yokohama, Japan) was compared with that of the nephrocytes. The rats were perfused (under anesthesia using pentobarbital) with physiological saline and subsequently $2.5 \%$ glutaraldehyde fixative that was buffered with $0.1 \mathrm{M}$ phosphate buffer. The fixed kidneys were further immersed in the same fixative and stored at $4{ }^{\circ} \mathrm{C}$.

\section{Conventional SEM}

Conventional SEM was performed as previously described (Dong et al. 2010). In brief, fixed samples were immersed in
$2 \%$ osmium tetroxide in $0.1 \mathrm{M}$ phosphate buffer for $2 \mathrm{~h}$. After dehydration using a graded series of ethanol, specimens were transferred to $t$-butyl alcohol and freeze-dried with an ES-2030 freeze dryer (Hitachi High-Technologies, Tokyo, Japan). After mounting on aluminum stubs with carbon paste, the dried specimens were coated with osmium using an OPC80T osmium plasma coater (Filgen, Nagoya, Japan) and observed using an S-4800 field-emission SEM (Hitachi High-Technologies).

\section{Sample preparation for FIB/SEM}

The fixed samples were processed using a combinatorial heavy metal staining protocol. This protocol was designed to enhance the signal for the backscatter electron imaging of epoxy-resin-embedded mammalian tissue at low accelerating voltages. In brief, the samples were successively immersed in $1 \%$ osmium tetroxide that contained $1.5 \%$ potassium ferrocyanide in $0.1 \mathrm{M}$ cacodylate buffer for $1 \mathrm{~h}$ on ice, $1 \%$ low molecular weight tannic acid (Electron Microscopy Sciences, Hatfield, PA) in $0.1 \mathrm{M}$ cacodylate buffer for $4 \mathrm{~h}$ at $25^{\circ} \mathrm{C}, 2 \%$ aqueous osmium tetroxide for $30 \mathrm{~min}$ at $25^{\circ} \mathrm{C}$ and $1 \%$ aqueous uranyl acetate overnight at $25^{\circ} \mathrm{C}$. The samples were then dehydrated with a graded series of ethanol and were embedded in epoxy resin, Oken Epok 812 (Okenshoji, Tokyo, Japan).

\section{FIB/SEM tomography}

Serial FIB/SEM images were obtained at 30-nm increments with a backscattered electron detector at a $2.0-\mathrm{kV}$ acceleration voltage using a Helios Nanolab 660 FIB/SEM (Thermo Fisher Scientific, Waltham, MA, USA). The pixel size of each FIB/ SEM image was $13.5 \times 17.1 \times 50 \mathrm{~nm} /$ pixel (width $\times$ height $\times$ depth). The pixel dimensions for a recorded image was $3072 \times 2048$ pixels. Thus, the dimension of serial imaging by FIB/SEM was $41.5 \times 35.0 \times 20.0-35.0 \mu \mathrm{m}$ (width $\times$ height $\times$ depth). The new surface for serial FIB/SEM imaging was generated by FIB milling using a $0.77-n A$ beam current, where gallium ions were accelerated with a voltage of $30 \mathrm{kV}$.

\section{Data processing for 3D reconstruction}

The 3D reconstruction of podocytes was performed using AMIRA 6.1 Software (Thermo Fisher Scientific) on a MousePro-W994DQP5X professional workstation (Mouse Computer Co. Ltd., Tokyo, Japan). We used a Cintiq 27QHD interactive pen display for the segmentation procedure (Wacom, Tokyo, Japan).

\section{Statistical analysis}

Width of foot processes and slit diaphragms were measured on FIB/SEM images. All measurements were shown as mean \pm 
Fig. 3 Sectional focused-ion beam/scanning electron microscope (FIB/ SEM) image of pericardial nephrocytes (PCNs) in adult Drosophila melanogaster. The contrast-inverted FIB/SEM images achieve a quality comparable to conventional transmission electron microscope (TEM) images. (a) PCN is localized near the heart tube in the dorsal hemolymph space (HL). CL, cardiac lumen; CW, cardiac wall. (b) Numerous electron-dense lysosomes in PCN. (c) Foot processes and slit diaphragms bridging them. Arrowheads, basement membrane of PCN. Scale bars: $1 \mu \mathrm{m}(\mathbf{a}) ; 500 \mathrm{~nm}(\mathbf{b}) ; 200 \mathrm{~nm}(\mathbf{c})$

SEM. Statistical analyses were performed using a two-tailed Student's $t$ test. $P$ values $<0.05$ were considered statistically significant.

\section{Results}

Drosophila melanogaster possesses two kinds of nephrocytes: garland and pericardial, in both its larval and adult stages (Zhuang et al. 2009). The garland and pericardial nephrocytes are located around the junction of the esophagus and proventriculus and along both sides of the heart tube, respectively. In the present study, we examined the 3D ultrastructure of the pericardial nephrocytes (PCNs) in D. melanogaster.

\section{Sectional (FIB/SEM) images of PCNs}

We obtained serial FIB/SEM images of PCNs in migrating larvae and adults. The contrast-inverted FIB/SEM images achieved a quality that was comparable to conventional transmission electron microscopy (TEM) images (Figs 3 and S1). The FIB/SEM images showed PCNs located near the heart tube (Fig. 3a) and possessed numerous electron-dense endosomes and lysosomes (Fig. 3b), which corresponded to the separation function of toxic materials from hemolymph in PCNs. The FIB/ SEM images are also able to visualize numerous fine foot processes and slit diaphragms bridging between them, as demonstrated by previous TEM studies (Crossley 1972) (Fig. 3c).

\section{Reconstructed images of normal PCNs}

The basal surfaces of individual PCNs were completely covered by a basement membrane (arrowheads in Fig. 3b,c); thus, conventional SEM was not suitable to observe their foot processes (Fig. 2h). To overcome this problem and reveal the 3D structure of PCNs in migrating larvae and adults, we reconstructed the basal surface of PCNs using their serial FIB/SEM images (Figs 4-6; Movies S2 and S3).

\section{PCNs in migrating larvae}

The basal surfaces of reconstructed PCNs were observed to be occupied by the foot processes and the slit-like spaces between neighboring foot processes (Fig. 4a,d,e; Movie S2).
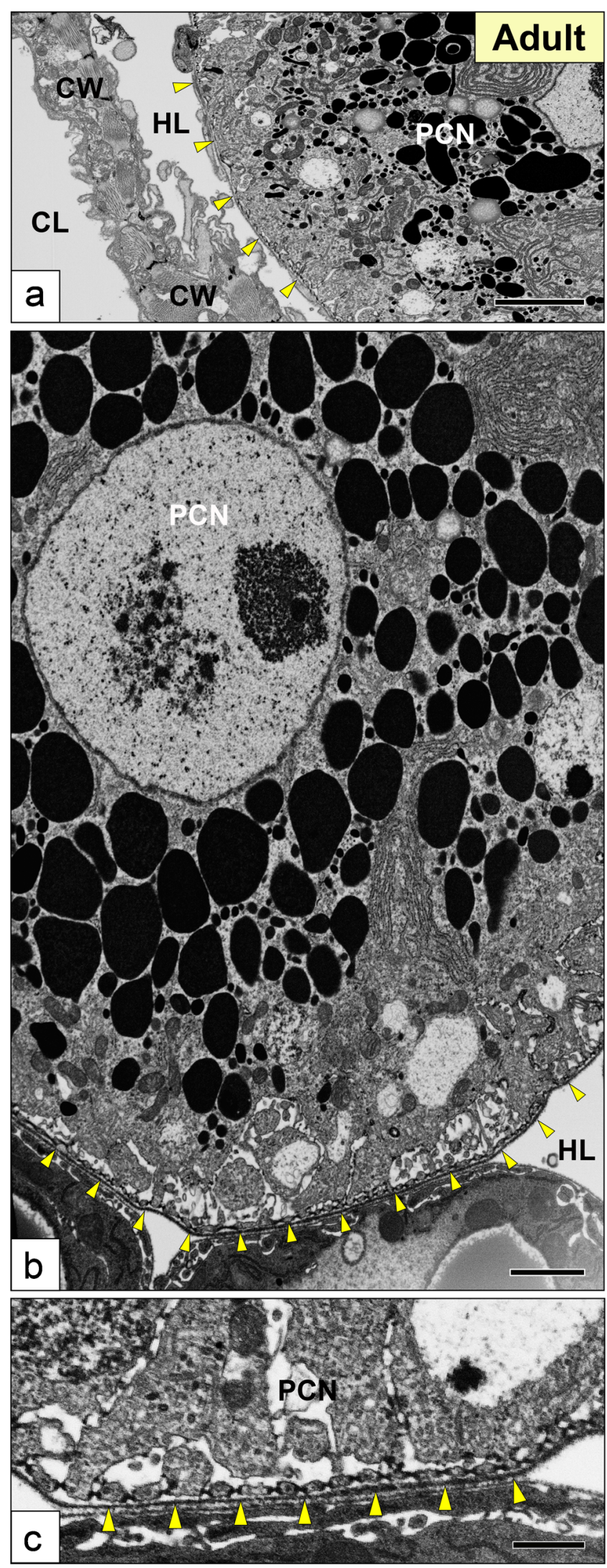

The slit-like spaces, which corresponded to the filtration slits in podocytes, were formed by the longitudinal infolding/ 

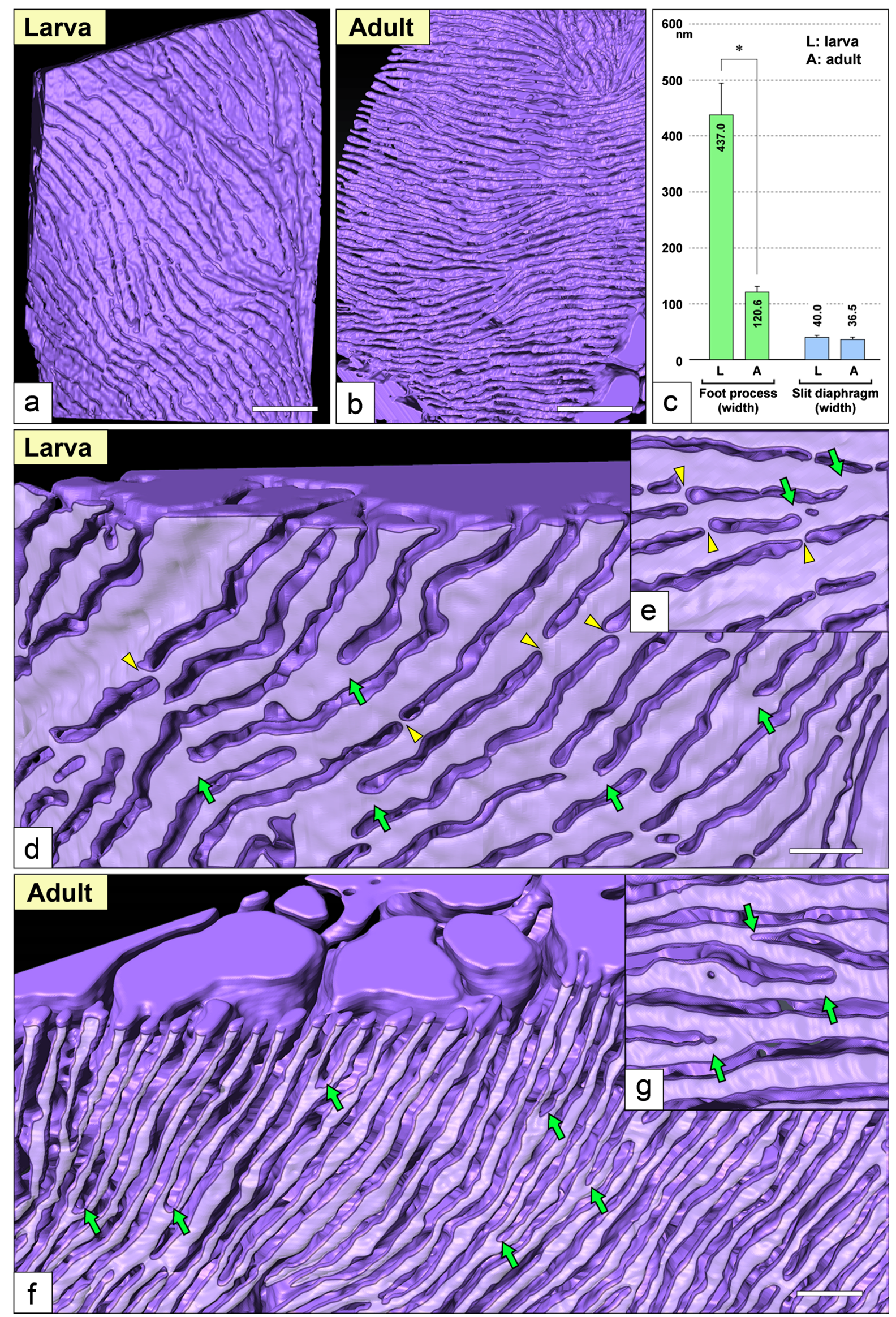
4 Fig. 4 Basal surface of reconstructed pericardial nephrocytes (PCNs) in Drosophila melanogaster. (a, d, e) PCN in migrating larva. (b, $\mathbf{f}, \mathbf{g}) \mathrm{PCN}$ in adult. $(\mathbf{a}, \mathbf{b})$ Total images of the basal surface of reconstructed PCNs. The basal surface, which has a "washboard-like" appearance, is clearly visible in the larva and adult reconstructed images. (d-g) Magnification of reconstructed images shown in (a) and (b). The basal surface membrane of foot processes, which adhere to the basement membrane, is shown in whitish purple. The foot processes run parallel to each other and their ends anastomose to the neighboring foot processes (green arrows in $\mathbf{d}-\mathbf{g}$ ). The thin cytoplasmic bridges are found between the neighboring foot processes in the larva (yellow arrowheads in $\mathbf{d}, \mathbf{e}$ ) but most of them were lost in the adult $(\mathbf{f}, \mathbf{g})$. The reconstructed PCNs shown here are also shown in Movies S2 and S3. Scale bars: $1 \mu \mathrm{m}(\mathbf{a}, \mathbf{b})$ and $500 \mathrm{~nm}(\mathbf{d}$, f). (c) Width of foot processes and slit diaphragms. The foot processes became significantly narrower in the adult than larval PCNs (green bars) but the width of the slit diaphragms was unchanged between the larva and adult (blue bars). Values represent means \pm standard errors of the means. Differences were tested using two-tailed Student's $t$ test. $* P<0.05$ was considered statistically significant

invagination of the basal plasma membrane (Fig. 4d). The remaining regions of the basal surface between the slit-like spaces became the foot processes.

Both ends of the foot processes were usually anastomosed to the neighboring foot processes (green arrows in Fig. 4d,e) and thus free ends were rarely observed. Furthermore, many tiny cytoplasmic bridges existed between the neighboring foot processes (yellow arrowheads in Fig. 4d,e; green lines in Fig. 5a). The slit diaphragm spanned the space surrounded by the foot processes and tiny cytoplasmic bridges. The foot processes almost continued to the main body of the $\mathrm{PCN}$ along their length and looked like "ridges" that protruded from the main body (Fig. 6a-c).

\section{PCNs in adults}

The foot processes of PCNs became significantly narrower in width in the adults than in the migrating larvae (Fig. 4b,f,g; green bars in Fig. 4c; Movie S3); however, the width of the slit diaphragms remained unchanged (blue bars in Fig. 4c). Moreover, the width of the foot processes was narrower in adult Drosophila PCNs than in the adult rat podocytes (Fig. 7a); however, the width of the slit diaphragm was similar between adult Drosophila PCNs and adult rat podocytes (Fig. $7 b)$.

Most of the tiny cytoplasmic bridges disappeared in adult PCNs; however, some of them remained (Figs $4 \mathrm{f}$ and $5 \mathrm{~b}$ ). The foot processes continued to the main body of the PCN only by several regions (Fig. 6d-h; Movie S4) and became thinner in comparison to those of larval PCNs as described above; they looked like "bars" (Fig. 6d-h; Movie S4).

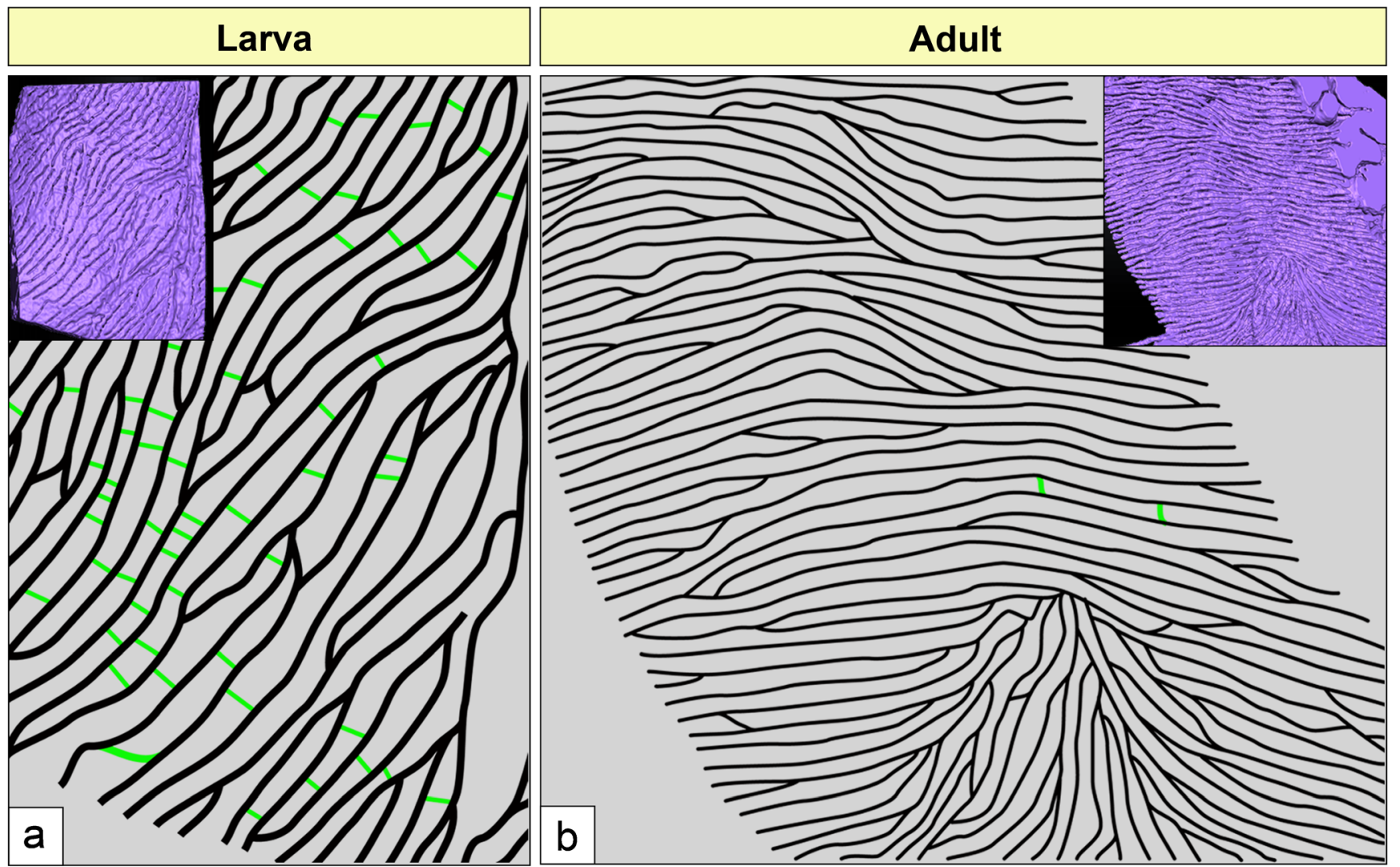

Fig. 5 Foot process arrangement of pericardial nephrocytes (PCNs) in Drosophila melanogaster. (a) PCN in migrating larva. (b) PCN in adult. The foot processes and tiny cytoplasmic bridges are depicted by black and green lines, respectively. Insets show the original reconstructed PCNs. Tiny cytoplasmic bridges are found between the neighboring foot processes in the larva (a) but most of them have disappeared in the adult (b) 


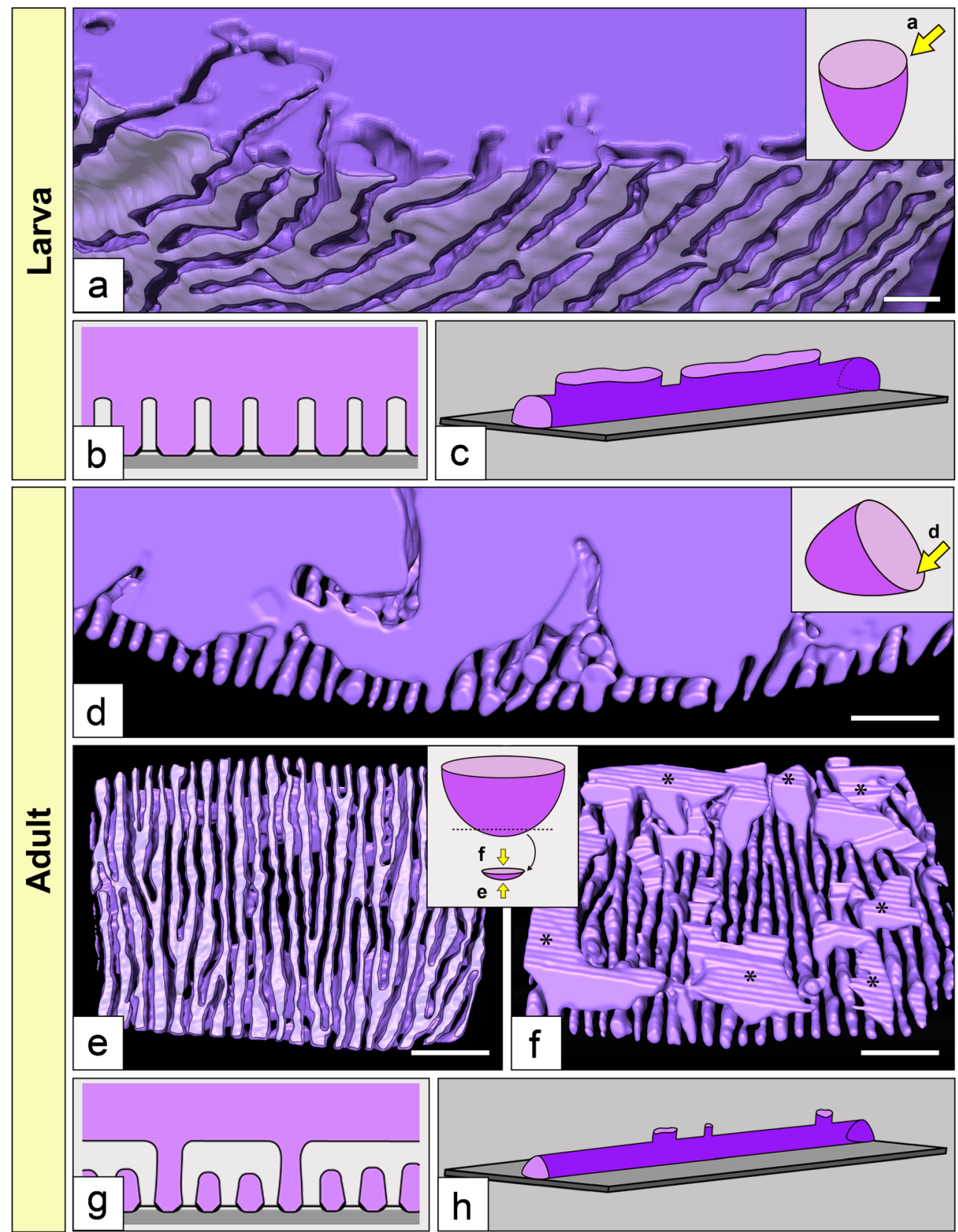

Fig. 6 Connection between the cell body and foot processes in pericardial nephrocytes (PCNs) in Drosophila melanogaster. (a-c) PCN in migrating larva. (a) Cross-section of reconstructed PCN. The direction of observation is indicated by an arrow in the inset. The slit-like spaces between the foot processes were formed by the longitudinal infolding/invagination of the basal plasma membrane. The foot processes were regarded as "ridgelike prominences" protruding from the cell body. The basal surface membrane attaching to the basement membrane is shown in whitish purple. (b) Schematic drawing showing the formation of foot processes. (c) Schematic drawing showing the connection between the cell body and foot processes. The foot processes almost continued to the main body of the PCN above as ridge-like prominences. (d-h) PCN in adult. (d) Cross-

\section{Reconstructed images of sns-knockdown PCNs}

FIB/SEM tomography was useful in revealing the normal 3D ultrastructure of PCNs. To confirm the usefulness of FIB/SEM section of reconstructed PCN. The direction of observation was indicated by an arrow in the inset. Unlike the larval PCNs, the foot processes lost the connection to the cell body over a wide area. (e, f) The foot processes sheared off from the cell body. The directions of observation were indicated by arrows in the inset. (e) The basal view. The basal surface membrane attaching to the basement membrane is shown in whitish purple. (f) The luminal view. The foot processes continued to the cell body only by several spots (asterisks) - therefore the foot processes exhibited a "barlike shape" in the adult PCNs. The reconstructed foot processes in (e) and (f) are also shown by Movie S4. (b4, b5) Schematic drawing showing the disconnection between the cell body and foot processes. Scale bars: $500 \mathrm{~nm}$

tomography in studying pathologic alterations of nephrocytes, we examined the 3D ultrastructure of sticks and stones (sns)knockdown PCNs using this method. Sns is a Drosophila ortholog of vertebrate $N P H S 1$, which encodes Nephrin, an 
400

$\mathrm{nm}$

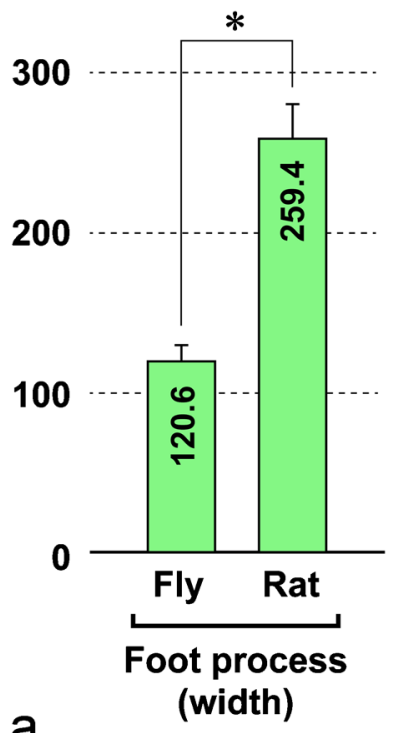

100

$\mathrm{nm}$

75

50

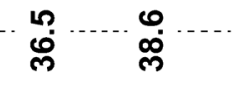

25

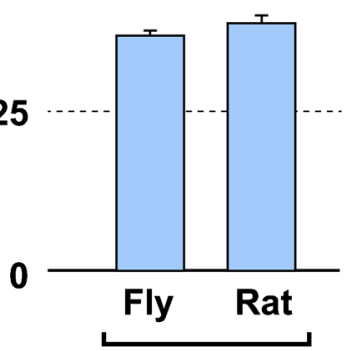

b

Slit diaphragm

(width)

Fig. 7 Quantitative comparison of Drosophila melanogaster pericardial nephrocytes (PCNs) and rat podocytes. (a) The width of the foot process was statistically significantly narrower in adult fly PCNs than in adult rat podocytes. (b) The width of the slit diaphragm was similar between fly and rat. Values are means \pm standard error of the mean. Differences were tested using two-tailed Student's $t$ test. $* P<0.05$ was considered statistically significant

essential component of the slit diaphragm in vertebrate podocytes (Gerth et al. 2005; Ichimura et al. 2013; Kestila et al. 1998; Kramer-Zucker et al. 2005).

The reconstructed images clearly showed the 3D structural alterations in sns-knockdown PCNs (Fig. 8; Movie S5). Compared to the control PCNs (Fig. 8c), the foot processes were irregular in width and shape and the slit-like spaces were predominantly reduced in sns-knockdown PCNs (Fig. 8a). Similar to control PCNs, the slit diaphragms were formed at the reduced slit-like spaces in sns-knockdown PCNs (Fig. 8b, d).

\section{Discussion}

The two-dimensional ultrastructure of nephrocytes has been investigated in numerous species of insects by using conventional TEM (Crossley 1984). However, the 3D ultrastructure of nephrocytes has not been precisely elucidated because the basement membrane that completely surrounds the individual nephrocytes makes it difficult to observe their 3D ultrastructure using conventional SEM. In the present study, FIB/SEM tomography, including a reconstruction technique, presented a viable solution to this problem and enabled the 3D ultrastructure of Drosophila PCNs to be analyzed, which has positive implications for further study of health and disease.

Similarity between PCNs and renal epithelial cells

Drosophila PCNs possess structural or functional similarities to some kinds of epithelial cells of the vertebrate nephron. Structurally, PCNs possess foot processes that link with the slit diaphragms like podocytes and have basal infoldings/ invaginations like the principal cells of the cortical collecting duct (Kaissling and Kriz 1979; Fukudome 2001). Functionally, PCNs absorb hemolymph proteins via the Drosophila orthologs of mammalian Cubilin and Amnionless (major receptors for protein reabsorption), like the proximal tubular epithelial cells (Zhang et al. 2013).

In invertebrate eucoelomates, the podocytes possess numerous lysosomes, including inclusion bodies (Ichimura and Sakai 2017), which indicates that the invertebrate podocytes serve to maintain the quality control of plasma proteins and to remove toxic substances from the blood, like insect nephrocytes. In other words, it can be argued that insect nephrocytes have retained some functional features of invertebrate podocytes.

\section{Nephrocytes in other invertebrate species}

Nephrocytes are recognized in three invertebrate phyla; Arthropoda, Onycophora and Mollusca (Crossley 1984; Haszprunar 1996; Seifert and Rosenberg 1977). The twodimensional ultrastructure of nephrocytes has been reported in a variety of species belonging to these phyla (Boer and Sminia 1976; Goodman and Cavey 1990; Kokkinopoulou et al. 2014). In several kinds of insects and decapods, nephrocytes have been reported to possess multiple nuclei (Crossley 1984). However, it is unclear whether such nephrocytes possess multiple nuclei per cell, as is the case in the garland nephrocytes found in D. melanogaster (Zhuang et al. 2009), or if multiple nephrocytes are connected to form an epithelioid mass. Our research group has been revealing a great diversity in the $3 \mathrm{D}$ ultrastructure of nephrocytes among arthropod species, including insects, using FIB/SEM tomography and these data will be published in a subsequent research paper.

\section{Adaptation of FIB/SEM tomography to podocyte-related cells}

Almost all metazoans produce primary urine by ultrafiltration of body fluid via the filtration epithelium (Ichimura and Sakai 2017; Ruppert and Smith 1988; Ruppert et al. 2003; SchmidtRhaesa 2007). The acoelomates and pseudocoelomates, which do not possess a body cavity lined with mesothelium, generally use the terminal cells as the filtration epithelium (Wilson and Webster 1974). As is the case with nephrocytes, it is difficult to evaluate the 3D ultrastructure of terminal cells by conventional SEM because the terminal cells are almost completely enwrapped by the basement membrane. For 
Fig. 8 Structural disorganization of sticks and stones (sns)knockdown pericardial nephrocytes (PCNs) in Drosophila melanogaster. $(\mathbf{a}, \mathbf{c})$ Reconstructed images. The snsknockdown PCNs were produced by mating male $s n s$-UAS-RNAi (P $\{$ KK108577\} VIE-260B) and female Dot-GAL4. The foot processes were irregular in shape and width in the sns-knockdown larval PCN (a), in comparison to the control Dot-GAL4 larval PCN (c). The basal surface membrane attached to the basement membrane is shown in whitish purple. The reconstructed PCNs shown here are also shown in Movie S5. (b, d) FIB/SEM images. In the snsknockdown PCN, the labyrinths were simpler, although the slit diaphragms (arrowheads) were formed at the slit-like spaces similar to the control Dot-GAL4 PCN. Scale bars: $500 \mathrm{~nm}(\mathbf{a}, \mathbf{c})$; $100 \mathrm{~nm}(\mathbf{b}, \mathbf{d})$
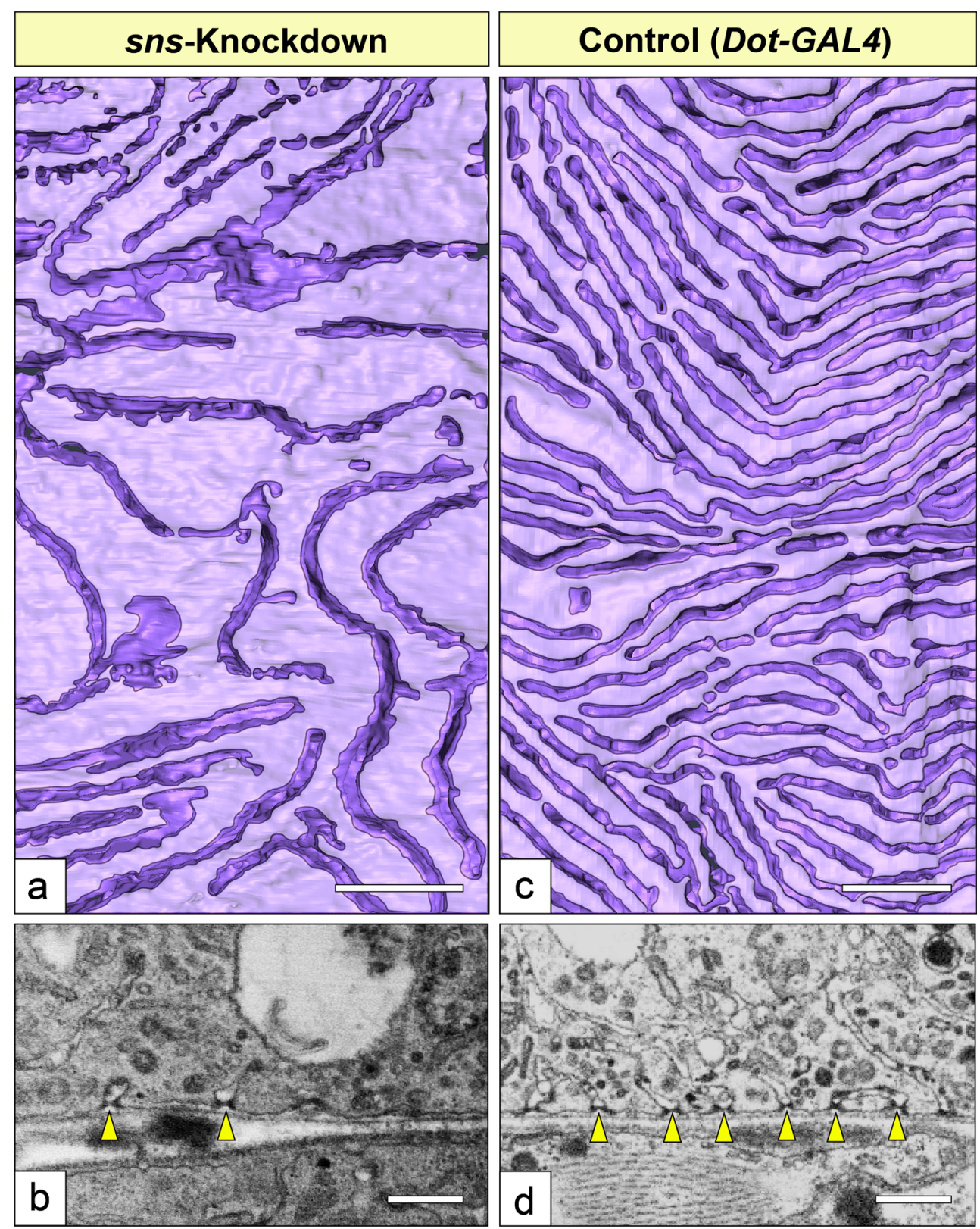

instance, in freshwater planarians, Ishii (1980) suggested that the filtration slits are formed between terminal cells, whereas another study reported that the filtration slits are formed as numerous rectangular fenestrations that open on the tubular cytoplasmic wall of the terminal cells (McKanna 1968). FIB/ SEM tomography should be useful in revealing the precise and accurate $3 \mathrm{D}$ architecture of terminal cells.

\section{Limitations of FIB/SEM tomography}

FIB/SEM tomography is a powerful tool for precisely analyzing the $3 \mathrm{D}$ ultrastructure of nephrocytes and podocytes; however, there are several technical disadvantages to this method. One of the major technical difficulties is that the observational volume is restricted; i.e., to smaller than approximately $40 \times 40 \times 40 \mu \mathrm{m}$ (width $\times$ height $\times$ depth). Therefore, it is impossible to obtain serial FIB/SEM images that contain the entire volume of one nephrocyte, podocyte, or glomerulus. Array tomography, which is used to obtain serial images from serial ultrathin sections mounted on silicon or glass bases, is a potential solution to this problem (Koga et al. 2018; Koike et al. 2017; Micheva and Smith 2007; Wacker and Schroeder 2013). This method enables serial images of larger volumes than FIB/SEM tomography to be obtained, which may enable the entire volume of the murine glomerular capillary to be reconstructed by array tomography (Dittmayer et al. 2018). 


\section{Conclusion}

FIB/SEM tomography is a powerful tool that can be used to reveal the $3 \mathrm{D}$ architecture of nephrocytes in more detail than previously possible using conventional TEM and SEM.

Acknowledgments The authors thank Mr. Takanobu Ishimura (Maxnet Co., Ltd., Tokyo, Japan) for the technical lecture on Amira software. The sns-UAS-RNAi (P \{KK108577\} VIE-260B) and Dot-GAL4 strains were obtained from the Vienna Drosophila RNAi Center and the Bloomington Drosophila Stock Center, respectively.

Author contributions K.I. designed the experiments. K.I. and S.K. obtained the serial FIB/SEM images. A.M. performed fly bleeding and knockdown experiments. Y.K., M.K., T.M. and K.I. performed threedimensional reconstruction. Y.K., T.S. and K.I. analyzed the experimental data. K.I. and Y.K. prepared the figures and wrote the main manuscript text. All authors reviewed the manuscript.

Funding This work was supported in part by the Japan Society for the Promotion of Science (JSPS) KAKENHI (grant nos. JP15K18960, JP17K08521 to K.I.) and the Foundation of Strategic Research Projects in Private Universities from the Ministry of Education, Culture, Sports, Science, and Technology of Japan (MEXT) (grant nos. S1311011, S1101009 to Juntendo University).

\section{Compliance with ethical standards}

Competing interests The authors declare there are no competing financial interests.

Ethical approval Animal experiments in this study were approved by the Institutional Animal Care and Use Committee of Juntendo University School of Medicine (approval no. 300226) and were carried out in accordance with the Guidelines for Animal Experimentation of Juntendo University School of Medicine.

Informed consent Not applicable.

Open Access This article is distributed under the terms of the Creative Commons Attribution 4.0 International License (http:// creativecommons.org/licenses/by/4.0/), which permits unrestricted use, distribution, and reproduction in any medium, provided you give appropriate credit to the original author(s) and the source, provide a link to the Creative Commons license, and indicate if changes were made.

\section{References}

Boer HH, Sminia T (1976) Sieve structure of slit diaphragms of podocytes and pore cells of gastropod molluscs. Cell Tissue Res 170:221-229

Crossley AC (1972) The ultrastructure and function of pericardial cells and other nephrocytes in an insect: Calliphora erythrocephala. Tissue Cell 4:529-560

Crossley AC (1984) Chapter 12. Nephrocytes and pericardial cells. In: Integument, respiration and circulation, 1 st ed: Pergamon Press. pp 487-515

Dittmayer C, Volcker E, Wacker I, Schroder RR, Bachmann S (2018) Modern field emission scanning electron microscopy provides new perspectives for imaging kidney ultrastructure. Kidney Int 94: 625-631

Dong HM, Ichimura K, Sakai T (2010) Structural organization of hepatic portal vein in rat with special reference to musculature, intimal folds, and endothelial cell alignment. Anat Rec 293:1887-1895

Fu Y, Zhu JY, Richman A, Zhang Y, Xie X, Das JR, Li J, Ray PE, Han Z (2017) APOL1-G1 in nephrocytes induces hypertrophy and accelerates cell death. J Am Soc Nephrol 28:1106-1116

Fukudome H (2001) A combined SEM and TEM study on the basal labyrinth of the collecting duct in the rat kidney. Arch Histol Cytol 64:339-351

Gerth VE, Zhou X, Vize PD (2005) Nephrin expression and threedimensional morphogenesis of the Xenopus pronephric glomus. Dev Dyn 233:1131-1139

Goodman SH, Cavey MJ (1990) Organization of a phyllobranchiate gill from the green shore crab Carcinus maenas (Crustacea, Decapoda). Cell Tissue Res 260:495-505

Haszprunar G (1996) The molluscan rhogocyte (pore-cell, Blasenzelle, cellule nucale), and its significance for ideas on nephridial evolution. J Molluscan Stud 62:185-211

Helmstädter M, Simons M (2017) Using Drosophila nephrocytes in genetic kidney disease. Cell Tissue Res 369:119-126

Ichimura K, Fukuyo Y, Nakamura T, Powell R, Sakai T, Janknecht R, Obara T (2013) Developmental localization of nephrin in zebrafish and medaka pronephric glomerulus. J Histochem Cytochem 61: 313-324

Ichimura K, Kakuta S, Kawasaki Y, Miyaki T, Nonami T, Miyazaki N, Nakao T, Enomoto S, Arai S, Koike M, Murata K, Sakai T (2017) Morphological process of podocyte development revealed by blockface scanning electron microscopy. J Cell Sci 130:132-142

Ichimura K, Miyaki T, Kawasaki Y, Kinoshita M, Kakuta S, Sakai T (2019) Morphological processes of foot process effacement in puromycin aminonucleoside nephrosis revealed by FIB/SEM tomography. J Am Soc Nephrol 30:96-108

Ichimura K, Miyazaki N, Sadayama S, Murata K, Koike M, Nakamura K, Ohta K, Sakai T (2015) Three-dimensional architecture of podocytes revealed by block-face scanning electron microscopy. Sci Rep 5:8993

Ichimura K, Sakai T (2017) Evolutionary morphology of podocytes and primary urine-producing apparatus. Anat Sci Int 92:161-172

Ishii S (1980) The ultrastructure of the protonephridial flame cell of the freshwater planarian Bdellocephala brunnea. Cell Tissue Res 206: 441-449

Kaissling B, Kriz W (1979) Structural analysis of the rabbit kidney. Adv Anat Embryol Cell Biol 56:1-123

Kestila M, Lenkkeri U, Mannikko M, Lamerdin J, McCready P, Putaala H, Ruotsalainen V, Morita T, Nissinen M, Herva R, Kashtan CE, Peltonen L, Holmberg C, Olsen A, Tryggvason K (1998) Positionally cloned gene for a novel glomerular proteinnephrin - is mutated in congenital nephrotic syndrome. Mol Cell $1: 575-582$

Kimbrell DA, Hice C, Bolduc C, Kleinhesselink K, Beckingham K (2002) The Dorothy enhancer has Tinman binding sites and drives hopscotch-induced tumor formation. Genesis 34:23-28

Koga D, Kusumi S, Watanabe T (2018) Backscattered electron imaging of resin-embedded sections. Microscopy 67:196-206

Koike T, Kataoka Y, Maeda M, Hasebe Y, Yamaguchi Y, Suga M, Saito A, Yamada H (2017) A device for ribbon collection for array tomography with scanning electron microscopy. Acta Histochem Cytochem 50:135-140

Kokkinopoulou M, Guler MA, Lieb B, Barbeck M, Ghanaati S, Markl J (2014) 3D-ultrastructure, functions and stress responses of gastropod (Biomphalaria glabrata) rhogocytes. PLoS One 9:e101078

Kolosov D, Tauqir M, Rajaruban S, Piermarini PM, Donini A, O'Donnell MJ (2018) Molecular mechanisms of bi-directional ion transport in 
the Malpighian tubules of a lepidopteran crop pest, Trichoplusia ni. J Insect Physiol 109:55-68

Kramer-Zucker AG, Wiessner S, Jensen AM, Drummond IA (2005) Organization of the pronephric filtration apparatus in zebrafish requires Nephrin, Podocin and the FERM domain protein mosaic eyes. Dev Biol 285:316-329

Kriz W, Kaissling B (2007) Structural organization of the mammalian kidney. In: Alpern RJ, Hebert SC, editors. Seldin and Giebisch's the kidney: physiology and pathophysiology, 4th ed: Academic Press. pp 479-563

McKanna JA (1968) Fine structure of the protonephridial system in planaria I. Flame cells. Z Zellforsch Mikrosk Anat 92:509-523

Micheva KD, Smith SJ (2007) Array tomography: a new tool for imaging the molecular architecture and ultrastructure of neural circuits. Neuron 55:25-36

Na J, Sweetwyne MT, Park AS, Susztak K, Cagan RL (2015) Dietinduced podocyte dysfunction in Drosophila and mammals. Cell Rep 12:636-647

Pavenstadt H, Kriz W, Kretzler M (2003) Cell biology of the glomerular podocyte. Physiol Rev 83:253-307

Ruppert E, Fox R, Barnes R (2003) Invertebrate zoology: a functional evolutionary approach, 7 th edn. Thomson Learning, Belmont

Ruppert E, Smith P (1988) The functional organization of filtration nephridia. Biol Rev 63:231-258

Ruppert E (1994) Evolutionary origin of the vertebrate nephron. Am Zool $34: 542-553$

Schmidt-Rhaesa A (2007) The evolution of organ systems. In: Oxford Unversity Press

Seifert G, Rosenberg J (1977) Die Ultrastruktur der Nephrozyten von Peripatoides leuckarti (Saenger 1869) (Onychophora, Peripatopsidae). Zoomorphologie 86:169-181
Sun Q, Wu Y, Jonusaite S, Pleinis JM, Humphreys JM, He H, Schellinger JN, Akella R, Stenesen D, Kramer H, Goldsmith EJ, Rodan AR (2018) Intracellular chloride and scaffold protein Mo25 cooperatively regulate transepithelial ion transport through WNK signaling in the Malpighian tubule. J Am Soc Nephrol 29:1449-1461

Tutor AS, Prieto-Sanchez S, Ruiz-Gomez M (2014) Src64B phosphorylates dumbfounded and regulates slit diaphragm dynamics: Drosophila as a model to study nephropathies. Development 141: 367-376

Wacker I, Schroeder RR (2013) Array tomography. J Microsc 252:93-99

Weavers H, Prieto-Sanchez S, Grawe F, Garcia-Lopez A, Artero R, Wilsch-Brauninger M, Ruiz-Gomez M, Skaer H, Denholm B (2009) The insect nephrocyte is a podocyte-like cell with a filtration slit diaphragm. Nature 457:322-326

Weide T, Vollenbroker B, Schulze U, Djuric I, Edeling M, Bonse J, Hochapfel F, Panichkina O, Wennmann DO, George B, Kim S, Daniel C, Seggewiss J, Amann K, Kriz W, Krahn MP, Pavenstadt H (2017) Pals1 haploinsufficiency results in proteinuria and cyst formation. J Am Soc Nephrol 28:2093-2107

Wilson RA, Webster LA (1974) Protonephridia. Biol Rev Camb Philos Soc 49:127-160

Zhang F, Zhao Y, Chao Y, Muir K, Han Z (2013) Cubilin and amnionless mediate protein reabsorption in Drosophila nephrocytes. J Am Soc Nephrol 24:209-216

Zhuang S, Shao H, Guo F, Trimble R, Pearce E, Abmayr SM (2009) Sns and Kirre, the Drosophila orthologs of Nephrin and Neph1, direct adhesion, fusion and formation of a slit diaphragm-like structure in insect nephrocytes. Development 136:2335-2344

Publisher's note Springer Nature remains neutral with regard to jurisdictional claims in published maps and institutional affiliations. 